\title{
Molecular Self-Assembly Inhibition Leads to Basket-Shaped Cyclophane Formation with Chiral Dynamics
}

Wei Wang, ${ }^{\dagger}$ Alex D. Bain, ${ }^{\S}$ Li-Qiong Wang, ${ }^{*}$ Gregory J. Exarhos, ${ }^{*}$ and Alexander D. Q. Li*

Department of Chemistry, Washington State University, Pullman, WA 99164. ${ }^{\S}$ Department of Chemistry, McMaster University, Hamilton, ON, Canada L8S 4M1, ${ }^{*}$ Fundamental Science

Directorate, Pacific Northwest National Laboratory, Richland, Washington 99354
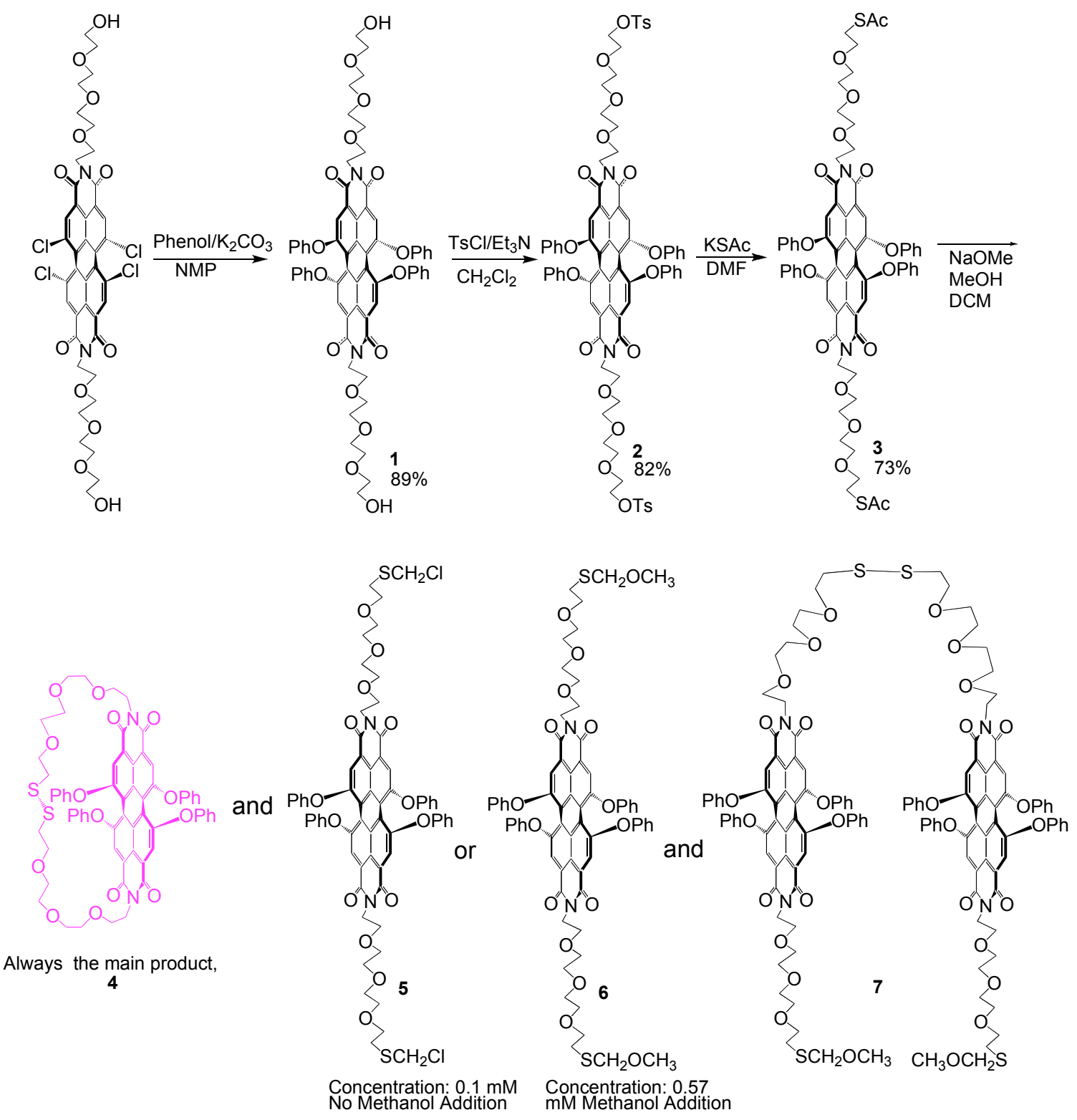

Supporting Figure 1. Synthetic route to the unimolecular perylene diimide cyclophane along with a few byproducts that were characterized. Note that linear dimer (7) was isolated but macrocylic dimer was not detected, indicating the formation of the linear dimer was mainly due to random collisions of two monomers rather than self-assembly effects. 


\section{GOESY Assignment}

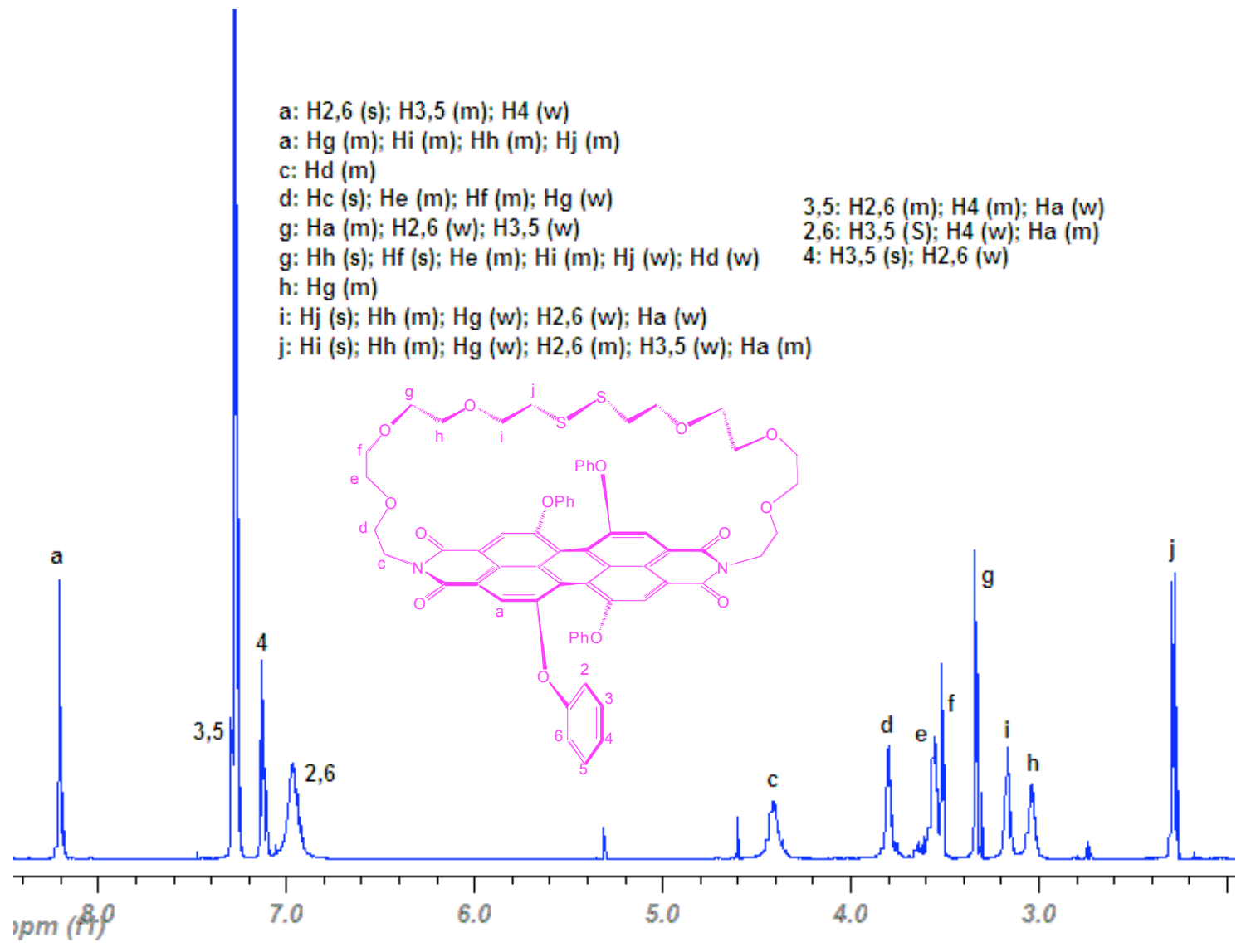

Supporting Figure 2. GOESY assignments indicate various degrees of interactions between adjacent protons. The interaction strengths are categorized into three levels, strong (s), medium $(\mathrm{m})$, and weak (w). 